\title{
Evaluación de ruido ambiental en alrededores a centros médicos de la localidad Barrios Unidos, Bogotá
}

\author{
Evaluation of environmental noise in the vicinity of medical centers in the Barrios \\ Unidos area, Bogotá
}

\section{Avaliação do ruído ambiental na vizinhança de centros médicos na localidade de Barrios Unidos, Bogotá}

Laura María Chaux-Álvarez

Baudilio Acevedo-Buitrago ${ }^{2}$

Recibido: octubre de 2018

Aceptado: abril de 2019

Para citar este artículo: Chaux-Álvarez, L. M; Acevedo-Buitrago, B. (2019). Evaluación de ruido ambiental en alrededores a centros médicos de la localidad Barrios Unidos, Bogotá. Revista Científica, 35(2), 234-246. Doi: https:// doi.org/10.14483/23448350.13983

\section{Resumen}

En este artículo se presentan los resultados de la evaluación de ruido ambiental en zonas aledañas a tres centros médicos ubicados en la localidad de Barrios Unidos (Bogotá). Esto con el objetivo de determinar el cumplimiento de los límites máximos permisibles para este tipo de sectores catalogados como de tranquilidad y silencio; para así verificar la influencia del desarrollo y crecimiento de la localidad en la potencial afectación a la salud de personas. La metodología desarrollada para los procesos de medición en los diferentes centros médicos está basada en los lineamientos dados en la Resolución n. ${ }^{\circ} 627$ del 2006 del Ministerio de Ambiente, Vivienda y Desarrollo Territorial. De esta manera, se realizó por cada centro médico la georreferenciación de la zona de estudio y mediciones preliminares, lo cual permitió establecer la existencia de contaminación por ruido y localizar un punto de mayor impacto en el que se hicieron las mediciones definitivas. De manera simultánea, se efectuó la toma de datos de condiciones meteorológicas, así como una caracterización de tráfico vehicular para construir mapas de ruido mediante el software SoundPlan. Los resultados obtenidos en cada centro médico demuestran, en general, que se sobrepasan los límites normativos de ruido ambiental (Leq A: 55dB), y que este fenómeno se correlaciona principalmente con el alto tránsito de vehículos, concurrencia de personas hablando y las actividades comerciales informales y formales que se han desarrollado de manera desordenada alrededor de la ubicación de los centros hospitalarios, convirtiendo a estos en un entornos comerciales y 
generando que no haya cumplimiento con relación al plan de ordenamiento establecido en la localidad.

Palabras clave: acústica, contaminación, georreferenciación, isofona, ruido ambiental.

\section{Abstract}

In the present project, environmental noise assessment is carried out in the vicinity of three medical centers located in the town of Barrios Unidos (Bogotá), with the objective of determining compliance with the maximum permissible limits for this type of sectors classified as tranquility and silence, and verify the influence of the development and growth of the locality on the potential impact on the health of people. The methodology developed for the measurement processes in the different medical centers is based on the guidelines given in Resolution 627 of 2006 of the Ministry of Environment. In this way, it was carried out by each medical center; georeferencing of the study area, and preliminary measurements, which allowed establishing the existence of noise pollution and locating a point of greater impact in which the definitive measurements were made. At the same time, meteorological data was taken, as well as a characterization of vehicular traffic to build noise maps using SoundPlan software. The results obtained in each medical center generally demonstrate that the regulatory limits of environmental noise are exceeded, and this phenomenon is mainly correlated with the high traffic of vehicles, the concurrence of people talking and the informal and formal commercial activities that have developed in a disorderly around the location of hospital centers, becoming a commercial environment and generating that there is no compliance with the ordering plan established in the locality. Keywords: acoustics, pollution, georeferencing, isophone, environmental noise.

\section{Resumo}

Neste projecto a avaliação do ruído ambiental em áreas circunvizinhas três centros médicos na cidade de Barrios Unidos (Bogotá) é realizada, a fim de determinar a conformidade com os limites máximos admissíveis para estes sectores classificados como tranquila e silêncio, e verificar a influência do desenvolvimento e crescimento da localidade sobre o impacto potencial sobre a saúde das pessoas. A metodologia desenvolvida para os processos de medição nos diferentes centros médicos é baseada nas diretrizes dadas na Resolução 627 de 2006 do Ministério do Meio Ambiente. Desta forma, foi realizado por cada centro médico; georreferenciamento da área de estudo, e medições preliminares, que permitiram estabelecer a existência de poluição sonora e localizar um ponto de maior impacto no qual as medidas definitivas foram feitas. Ao mesmo tempo, dados meteorológicos foram realizados, bem como uma caracterização do tráfego de veículos para construir mapas de ruído usando o software SoundPlan. Os resultados obtidos em cada centro médico geralmente mostram que limites regulatórios de ruído ambiente são excedidos, e esse fenômeno é principalmente correlacionadas com alto tráfego de veículos, multidão de pessoas conversando e atividades de negócios informais e formais que foram desenvolvidas para desordenada em torno da localização dos centros hospitalares, tornando-se um ambiente comercial e gerando que não há conformidade com o plano de encomenda estabelecido na localidade.

Palavras-chaves: acústica, poluição, georreferenciamento, isofone, ruído ambiental.

\section{Introducción}

Según Murphy y Rice, "El ruido se entiende como cualquier sonido no deseado o potencialmente dañino, que es generado por las actividades humanas y que deteriora la calidad de vida de las personas" (2009); o también se puede definir como un sonido, irritante, perturbador o molesto que interfiere con alguna actividad o con el descanso (Miyara, 1999). Este fenómeno acústico se considera como contaminante dado que produce diferentes afectaciones al ser humano, causando molestia, dificultad para conciliar el sueño, pérdida de calidad del sueño, dolor de cabeza, estrés, insomnio, hipertensión, discapacidad auditiva, trastornos gastrointestinales, etc.; además de problemas en la comunicación verbal. A la par que 
estos problemas, se genera un retraso en la recuperación de los pacientes hospitalizados (Stassen et al., 2008; Arana y García, 1998; Evans et al., 2001; Abbasi et al., 2018; Loupa et al., 2019). Es por todo esto que la Organización Mundial de la Salud (OMS) ha definido a la contaminación auditiva como el tercer problema ambiental de mayor relevancia en el mundo (OMS, 2008).

Durante los últimos años, en la ciudad de Bogotá se ha encontrado de manera generalizada problemas de contaminación por ruido en todo tipo de zonas, entre comerciales y residenciales, así como en sectores de tranquilidad (Pacheco, Franco y Behrentz, 2009), y se muestran las localidades de Kennedy, Engativá, Chapinero y Barrios Unidos como las más afectadas (Alcaldía Mayor de Bogotá, 2014).

En la localidad de Barrios Unidos se presenta el mayor número de quejas por contaminación de ruido. De igual modo, en el Plan Ambiental Local de la localidad de Barrios Unidos se pudo identificar que, en el periodo diurno y nocturno, se encuentran niveles de ruido en el intervalo de 75 dB a $80 \mathrm{~dB}$ ligados a la alta influencia de actividades como: funcionamiento de talleres automotrices, establecimientos comerciales y diferentes tipos de ventas ambulantes, rutas que atraviesan el espacio aéreo de esta y tráfico automotor (De Barrios Unidos, 2017). En esta localidad están ubicados siete Unidades de Atención Primaria (UPA), un Centro de Atención Prioritaria en Salud (CAPS) y dos Hospitales, y para este tipo de establecimientos, dada su función específica así como para las zonas aledañas de ubicación, las directivas dadas en la normatividad de ruido en el mundo y a nivel nacional expresan que se deben considerar como sectores de tranquilidad y silencio; razón por la cual se tiene que garantizar su buen funcionamiento, ya que en estos sitios genera afectación en la salud de los pacientes causando agudización de los fenómenos que produce el ruido, así como trastornos en los procesos de recuperación (Acevedo, 2011; Alonso, 2003; Secretaria Distrital de Ambiente, 2009; Committee on Environmental Health, 1997; Gutiérrez, 2011; Jones, 2010).

Con el presente estudio se busca definir y documentar el cumplimiento de los estándares máximos permisibles de ruido ambiental, establecidos en la Resolución n. ${ }^{\circ} 627$ del 2006 del Ministerio de Ambiente, Vivienda y Desarrollo Territorial (MAVDT), en microambientes de zonas aledañas a tres centros de servicio médico ubicados en las UPZ 12 de octubre y los alcázares de la localidad de Barrios Unidos, determinar las causas y posibles fuentes de ruido y sus áreas de influencia y así definir acciones y alternativas que puedan mitigar el alto nivel de ruido.

\section{Metodología}

La realización de las actividades del trabajo propuesto estuvo guiada bajo los aspectos técnicos y metodológicos descritos en la norma ISO 1996-1 y la Resolución n. ${ }^{\circ}$ 627/06 del MAVDT. De esta manera, se seleccionaron como eje del trabajo centros de salud que han sido clasificados como sectores de tranquilidad y silencio. Las mediciones de ruido fueron hechas en el periodo comprendido entre enero 18 a febrero 11 de 2018, en jornada diurna, con un sonómetro tipo 1 marca y modelo Svantek 977, el cual posee una precisión de $\pm 1 \mathrm{~dB}$ en el rango de frecuencia $20 \mathrm{~Hz}$ a $20 \mathrm{kHz}$, con analizador en tiempo real de $1 / 3$ de octava, bajo intervalo de ponderación A y operado en modo rápido (con activación simultánea para medición también en impulsivo y lento). Las condiciones meteorológicas (velocidad del viento, dirección del viento, precipitación, temperatura, y humedad relativa) se midieron al mismo tiempo con una estación meteorológica marca Davis Pro (Davis Instruments, 2002).

\section{Medición preliminar y selección de puntos críticos}

Se realizaron mediciones preliminares en zonas aledañas a cada hospital con el fin de establecer 
que, primero, existe una problemática de ruido ambiental en las zonas cercanas al centro médico de estudio y, posteriormente, para precisar por cada hospital un punto crítico o sitio de mayor contaminación por ruido. En estos puntos, se realizaron las mediciones definitivas y llevadas a cabo por un mayor intervalo de tiempo, para de esta manera evidenciar si el problema y la influencia de las fuentes se mantiene constante durante el día de medición. Dichas mediciones se efectuaron durante 15 minutos por punto y simultáneamente se tomaron mediciones de las condiciones meteorológicas, así como también se efectúo la identificación y especificación de eventos generadores de ruido durante las mediciones. luego, se procedió a ejecutar una caracterización de la zona, identificando fuentes fijas a $300 \mathrm{~m}$ alrededor de cada centro médico y referenciándolas mediante el uso de GPS.

\section{Mediciones definitivas de ruido}

De acuerdo con los resultados de las mediciones preliminares en cada centro de salud, se seleccionó un punto de medición que presentó el peor escenario $y$, dependiendo de la jornada de funcionamiento, se estableció el horario de medición en periodo diurno de tres horas de tal manera que se evaluaron los niveles de presión sonora durante dos días. Un día entre semana en un horario de transito que cubre pico y placa y hora valle, y un día en fin de semana, en el mismo horario y teniendo en cuenta que en este día no hay pico y placa. De manera simultánea, se realizaron mediciones de condiciones meteorológicas, así como la identificación de eventos característicos generadores de ruido.

\section{Mapas de distribución de ruido}

Para cada hospital se determinó la influencia del ruido ambiental a través de la realización de mapas de distribución de ruido de manera asimétrica con relación al tiempo, por medio del software
SoundPlan, y con ayuda del programa Arcgis, así como de herramientas de georreferenciación como Google Earth; Se crearon diferentes isófonas en formas de contornos con el fin de establecer el comportamiento de propagación y los niveles de presión sonora en las zonas aledañas a los centros de salud de estudio.

\section{Resultados}

\section{Medición preliminar y selección de puntos críticos}

\section{Resultados preliminares Hospital Universitario Barrios Unidos}

De acuerdo con la georreferenciación y caracterización de la zona de influencia alrededor del centro médico, se puede apreciar que una de las principales fuentes de ruido está dada por actividades asociadas al alto tráfico vehicular en las calles secundarias, las cuales se encuentran habilitadas para el acceso de ambulancias y vehículos hacia el hospital, alimentadas a su vez por la vía principal anexa de la calle 68. Se observa también que existen establecimientos de actividades comerciales ubicados en las cercanías al hospital, tales como restaurantes y un centro comercial, los que por su menor distancia al hospital pueden tener mayor incidencia en la generación de ruido; actividad comercial informal, actividades académicas en colegio y transeúntes hacia parqueaderos, y hacia una iglesia que dado su horario de funcionamiento provee ruido en tiempos específicos. De esta manera, se identifican dos puntos de alta influencia de ruido ambiental: la entrada principal al hospital y un punto cercano a la iglesia, en los cuales se realizaron las mediciones preliminares de ruido. Los resultados se pueden observar en la figura 1.

De acuerdo con los resultados obtenidos, se puede establecer que los niveles de presión sonora (NPS) en los dos puntos de medición sobrepasan los límites permisibles para sectores de tranquilidad y silencio (55 Leq A dB, para el horario diurno). 


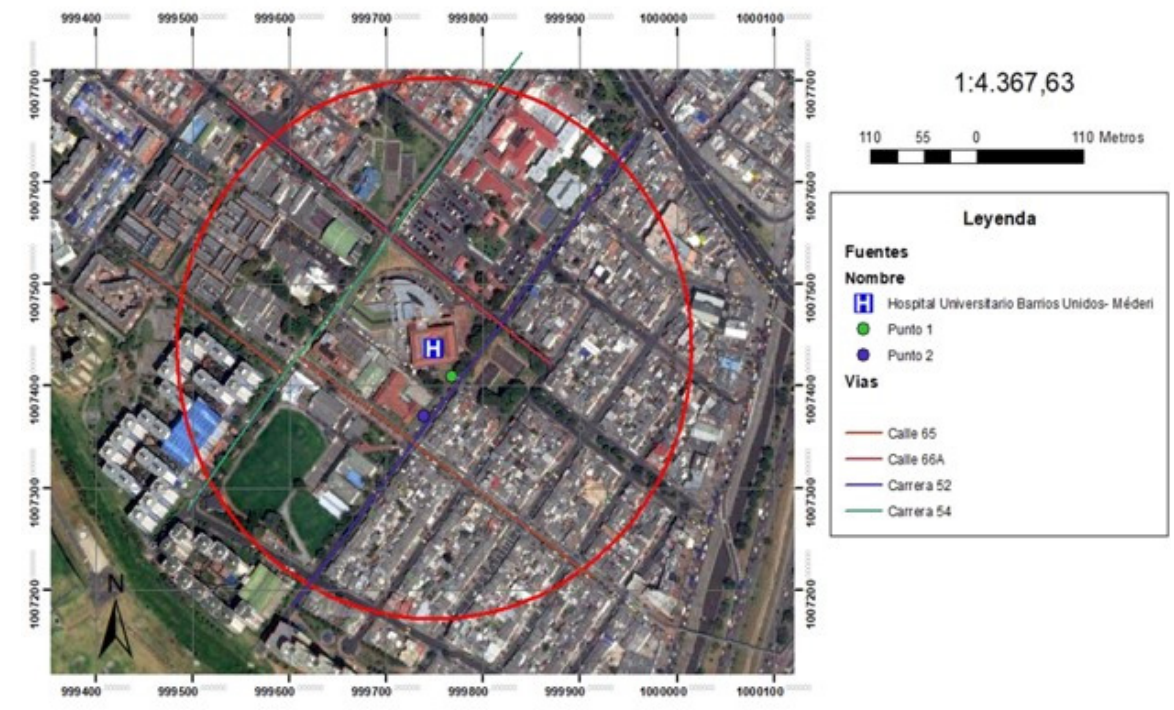

\begin{tabular}{|c|c|c|c|c|}
\hline Punto & Distancia $(\mathbf{m})$ & Actividades & Día/Hora & Leq A (dB) \\
\hline Punto \# 1 & 15,64 & $\begin{array}{c}\text { Flujo vehicular, paso de aviones, paso } \\
\text { de personas, puestos comerciales. }\end{array}$ & $\begin{array}{c}7 \text { de Julio } 2017 / 11: 10 \\
\text { a.m. }-11: 25 \text { a.m. }\end{array}$ & $68-72$ \\
\hline Punto \#2 & 44,81 & $\begin{array}{c}\text { Flujo vehicular, paso de aviones, paso } \\
\text { de personas, actividades de la iglesia }\end{array}$ & $\begin{array}{c}7 \text { de Julio 2017 / 10:40 } \\
\text { a.m. - 10:55 a.m. }\end{array}$ & $66-68$ \\
\hline
\end{tabular}

Figura 1. Ubicación de los puntos preliminares Hospital Barrios Unidos.

Fuente: elaboración propia.

Sin embargo, se observa un mayor intervalo de variabilidad y un mayor nivel de presión sonora en el punto 1 de medición con relación a las mediciones en el punto 2, esto marcado por la actividad comercial informal cercana a este punto.

\section{Resultados preliminares CAPS de Chapinero}

En los resultados de georreferenciación y caracterización se puede apreciar que las principales fuentes de ruido son: primero, las fuentes móviles dada la cercanía con la vía principal calle 66 que presenta alto flujo vehicular, siendo una vía de acceso importante para la localidad por su conexión a la avenida Caracas, se destaca también la proximidad del paradero de buses del sistema integrado de transportes (SITP) lo cual provee actividad específica relacionada con los transeúntes y el comercio informal asociado. Segundo, las fuentes fijas que rodean al centro médico, tales como establecimientos de metalurgia y ferretería, así como fabricación de tejados, grifería, instalaciones eléctricas y tornillos. Se localizaron dos puntos de alta influencia de ruido: la entrada principal al centro médico y la intersección de la calle 66 con la carrera 16, en los cuales se realizaron las mediciones preliminares de ruido ambiental. Los resultados se pueden observar en la figura 2 .

\section{Resultados preliminares Fundación Hospital In- fantil Universitario de San José}

En la caracterización de ubicación se puede observar que en este hospital se tiene una mayor influencia de ruido debido al alto tráfico vehicular que a las actividades comerciales, ya que al frente del hospital se encuentra la vía principal 
avenida calle 68, y muy cerca la avenida carrera 30. También se puede considerar como fuentes apreciables: talleres de mecánica y el tránsito de personas hacia supermercados, droguerías y panaderías. De acuerdo con lo anterior, se establecieron dos puntos de alta influencia de ruido: la entrada principal al centro médico y la entrada de urgencias, en los cuales se realizaron las mediciones preliminares de ruido ambiental. Los resultados se pueden observar en la figura 3.

De acuerdo con los resultados se puede establecer que los NPS en los dos puntos de medición sobrepasan los límites permisibles para el sector de tranquilidad y silencio, y se observa un mayor intervalo en los NPS obtenidos en la medición realizada en el punto 1.

\section{Resultados de las mediciones definitivas de ruido ambiental}

En la tabla 1 se observan los resultados promedio corregidos de las mediciones definitivas realizadas durante tres horas, en tres diferentes centros médicos tomados como referencia en la localidad de Barrios Unidos. De estos resultados se puede establecer que, para cada uno de los hospitales, y en el punto que mostraba el peor escenario se confirman los resultados ya presentados en las mediciones preliminares realizados en días diferentes a las mediciones definitivas, no se cumple con los límites máximos permisibles de ruido en horario diurno dados en la Resolución n. ${ }^{\circ} 627$ de 2006 del MAVDT, para sectores de tranquilidad y silencio en días entre semana y en fin de semana.

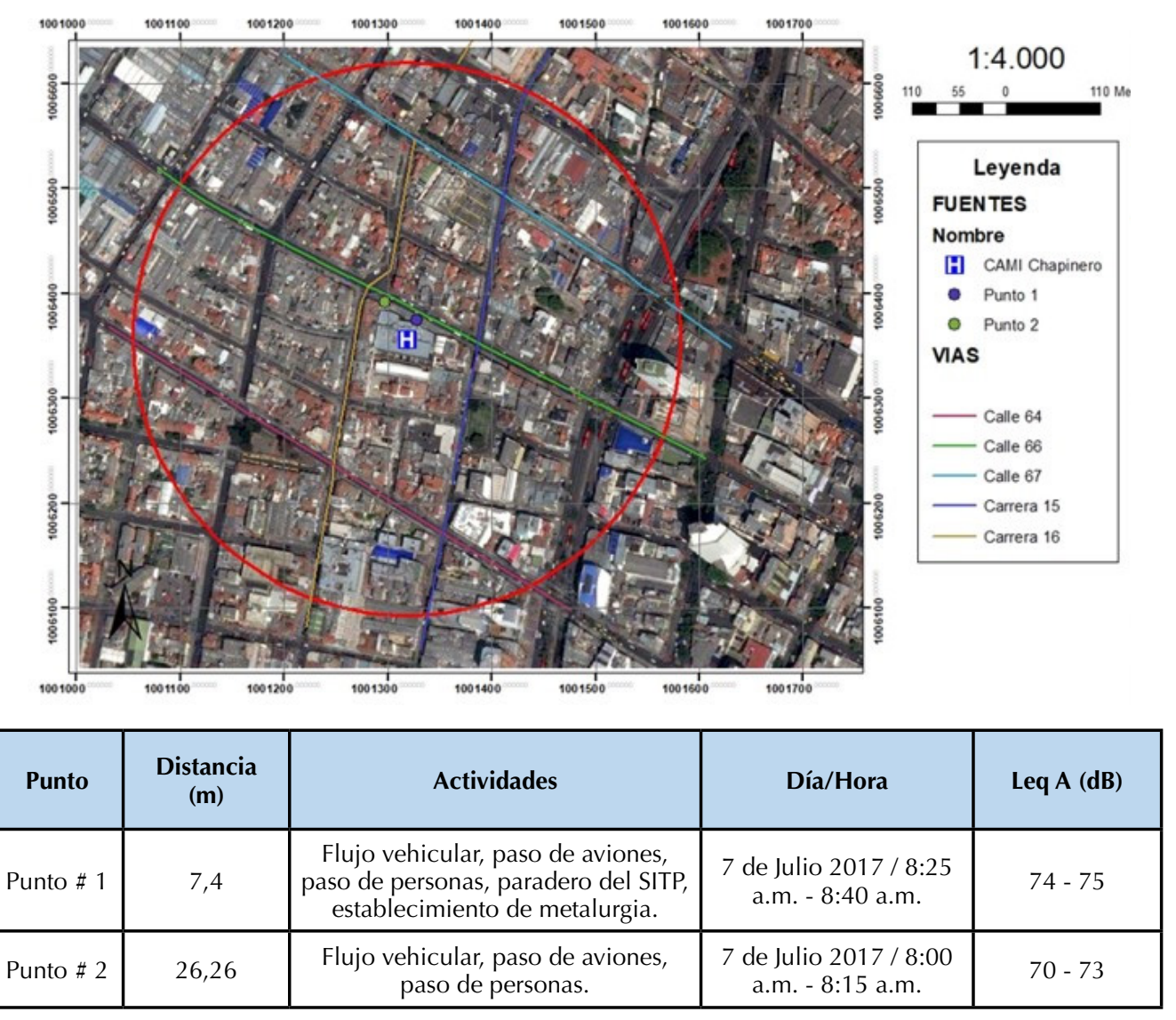

Figura 2. Ubicación de los puntos preliminares CAPS de Chapinero.

Fuente: elaboración propia. 

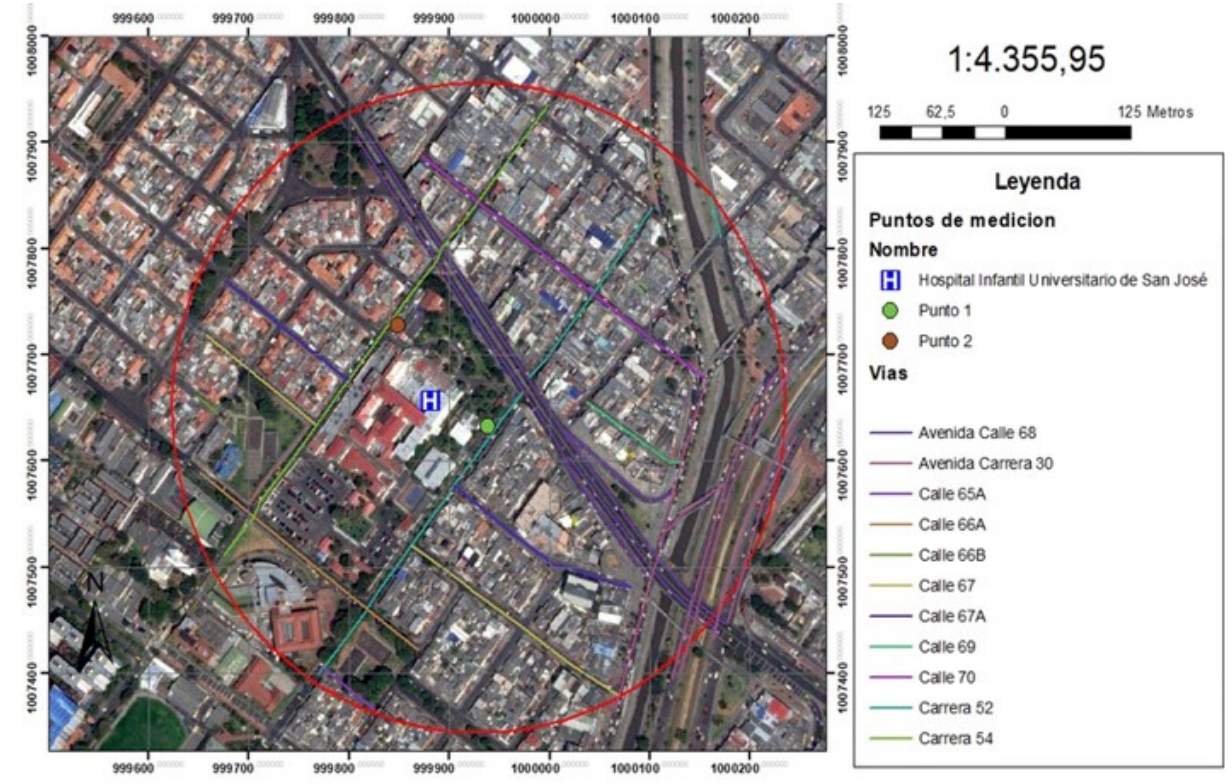

\begin{tabular}{|c|c|c|c|c|}
\hline Punto & $\begin{array}{c}\text { Distancia } \\
(\mathbf{m})\end{array}$ & Actividades & Día/Hora & Leq A (dB) \\
\hline Punto \# 1 & 45,57 & $\begin{array}{c}\text { Flujo vehicular, paso de aviones, } \\
\text { paso de personas. }\end{array}$ & $\begin{array}{c}7 \text { de Julio } 2017 / 2017 \\
9: 30 \text { a.m. }-9: 45 \text { a.m. }\end{array}$ & $72-74$ \\
\hline Punto \#2 & 56,15 & $\begin{array}{c}\text { Flujo vehicular, paso de aviones, } \\
\text { paso de personas. }\end{array}$ & $\begin{array}{c}7 \text { de Julio 2017 } / 9: 55 \\
\text { a.m. }-10: 10 \text { a.m. }\end{array}$ & $68-71$ \\
\hline
\end{tabular}

Figura 3. Ubicación de los puntos preliminares Fundación Hospital Infantil Universitario De San José.

Fuente: elaboración propia.

Tabla 1. Nivel de presión sonora promedio corregido para cada centro médico de estudio en la localidad de Barrios Unidos.

\begin{tabular}{|c|c|c|c|c|c|c|}
\hline Punto de medición & \begin{tabular}{|c|} 
Hospital \\
Universitario Barrios \\
Unidos
\end{tabular} & & $\begin{array}{l}\text { CAPS de } \\
\text { Chapinero }\end{array}$ & & $\begin{array}{c}\text { Fundación } \\
\text { Hospital Infantil } \\
\text { Universitario de } \\
\text { San Jose }\end{array}$ & \\
\hline Coordenadas & $\begin{array}{l}\text { Latitud } 4^{\circ} 39^{\prime} 48.83^{\prime \prime} \\
\mathrm{N} \quad \text { Longitud: } 74^{\circ} \\
4^{\prime} 47.83^{\prime \prime} \mathrm{O}\end{array}$ & & $\begin{array}{l}\text { Latitud } \\
4^{\circ} 39^{\prime} 13.38^{\prime \prime} \mathrm{N} \\
\text { Longitud: } 74^{\circ} \\
3^{\prime} 55.84^{\prime \prime} \mathrm{O}\end{array}$ & & $\begin{array}{l}\text { Latitud } \\
4^{\circ} 39^{\prime} 55.05^{\prime \prime} \mathrm{N} \\
\text { Longitud: } 74^{\circ} \\
4^{\prime} 40.74^{\prime \prime} \mathrm{O}\end{array}$ & \\
\hline Dias de medición & Miércoles & Sábado & Jueves & Domingo & Viernes & Sábado \\
\hline Hora de inicio & 8:00 & 8:00 & $7: 00$ & $7: 00$ & $7: 00$ & $7: 00$ \\
\hline Hora de finalización & 11:00 & 23:00 & 10:00 & 10:00 & 10:00 & 10:00 \\
\hline Calibracion inicial $L_{e q}(A) d B$ & 115.4 & 115.4 & 114.4 & 115.3 & 114.5 & 115.4 \\
\hline Calibracion final $L_{e q}(A) d B$ & 115.3 & 115.2 & 114.3 & 115.2 & 114.5 & 115.2 \\
\hline $\mathrm{L}_{\mathrm{eq}}(\mathrm{A}) \mathrm{dB}$ & 61.3 & 70.6 & 68.9 & 68.5 & 58.5 & 69.1 \\
\hline $\mathrm{L}_{90}(\mathrm{~A}) \mathrm{dB}$ & 62.2 & 70.4 & 67.9 & 69.5 & 59.9 & 69.4 \\
\hline
\end{tabular}

Fuente: elaboración propia. 
En ninguno de los casos en las mediciones en los diferentes hospitales se observa diferencia apreciable en los promedios horarios entre semana y su correlación con los eventos de tráfico vehicular (pico y placa y hora valle) ya que el tráfico vehicular es muy denso por la cercanía a vías principales y este no tiene una disminución apreciable de paso de vehículos; el cambio básicamente está dado por una mayor velocidad del paso de los mismos en la hora valle con relación a la hora pico. Además, mientras que para el CAPS Chapinero se evidencia un resultado promedio prácticamente similar del ruido medido el día entre semana y el fin de semana, para el caso del hospital universitario Barrios Unidos y el de la Fundación Hospital Infantil Universitario de San José, se presentan mayores NPS durante las mediciones realizadas en el día de fin de semana en los cuales no hay restricción de tráfico, y la cercanía a las avenidas principales o secundarias evidenció mediante conteo un mayor paso de vehículos en estos sitios.

En la tabla 2 se puede apreciar que las actividades y eventos característicos ligados al ruido ambiental en la zona del Hospital Universitario Barrios Unidos para las mediciones realizadas en el día entre semana presentan un intervalo entre $60.3 \mathrm{~dB}$ a 62.3 dB. Además, que están dados por tránsito aéreo y vehicular, el comercio informal, las actividades realizadas en la iglesia y el tránsito de personas hablando, lo cual se pudo verificar como una actividad constante; durante el fin de semana se aumenta este tipo de actividad comercial aunado al tránsito vehicular y uso de parqueaderos, por el incremento de visitas a los pacientes alojados en el hospital, así como a la mayor actividad registrada en los parques aledaños, lo cual hace que los NPS aumenten de manera significativa su intervalo de $70.5 \mathrm{~dB}$ a $71.1 \mathrm{~dB}$.

En el CAPS de Chapinero el intervalo de NPS estuvo entre $68 \mathrm{~dB}$ y $71 \mathrm{~dB}$ para el periodo de medición de ruido, y no se evidenció diferencia en cuanto a la hora pico de tránsito vehicular con relación a la hora valle, solo se ve un aumento de velocidad de los vehículos, el flujo de vehículos en la calle 66 es constante debido a la conexión que da a la avenida Caracas. Respecto a la incidencia de fuentes fijas se encontró que existe un establecimiento de metalurgia ubicado al frente del centro médico y con un horario de funcionamiento de 8 am a 5 pm, el cual provee una alta propagación del ruido de tipo impacto y fluctuante, dadas las actividades allí relacionadas (golpeteo con martillo, uso de equipos de soldadura, pulidora y cortadora) haciendo muy importante su influjo en las mediciones realizadas ya que aumenta el NPS promedio.

Para la Fundación Hospital Universitario de San José los resultados muestran que entre semana el intervalo de NPS estuvo entre $58.5 \mathrm{~dB}$ y $60.1 \mathrm{~dB}$ para el periodo de medición de ruido, los principales eventos están dados por el flujo vehicular en la avenida calle 68 y la conexión con la avenida carrera 30. En la medición de ruido para el fin de semana se encontró un intervalo de ruido más elevado: de $67 \mathrm{~dB}$ a $70 \mathrm{~dB}$, dando respuesta a los eventos y actividades que se realizan los fines de semana, como lo son el aumento de tránsito de vehículos por la no aplicación de la restricción por pico y placa y esto ligado a las visitas de personas al hospital, que a su vez incrementa el comercio informal, el número de personas hablando y uso del parqueadero. También se incrementa el uso de zonas de recreación como parques cercanos.

\section{Mapas de ruido}

\section{Mapa de distribución del Hospital Universitario Barrios Unidos}

A partir de la caracterización de las zonas aledañas al punto de interés, las mediciones realizadas, e incorporando una descripción del área (300 metros alrededor de cada centro médico), el modelo aplicó los efectos de la propagación del sonido. Según el mapa de ruido (figura 4), las áreas más críticas, sombreadas con el color azul, se ubican en las vías alrededor del centro médico y llegan 
Tabla 2. Nivel de presión sonora promedio por hora para cada centro médico de estudio en la localidad de Barrios Unidos.

\begin{tabular}{|c|c|c|c|c|c|c|c|c|}
\hline \multicolumn{3}{|c|}{ Hospital Universitario Barrios Unidos } & \multicolumn{3}{|c|}{ CAPS de Chapinero } & \multicolumn{3}{|c|}{$\begin{array}{l}\text { Fundacion Hospital Infantil } \\
\text { Universitario de San Jose }\end{array}$} \\
\hline Dia & Hora & $L_{e q} \mathrm{~A}(\mathrm{~dB})$ & Dia & Hora & $L_{\theta q} \quad \mathrm{~A}(\mathrm{~dB})$ & Dia & Hora & $L_{e q} \mathrm{~A}(\mathrm{~dB})$ \\
\hline \multirow{3}{*}{$\begin{array}{c}\text { Dia entre } \\
\text { semana }\end{array}$} & $8: 00-9: 00$ & 61.3 & \multirow{3}{*}{$\begin{array}{c}\text { Dia entre } \\
\text { semana }\end{array}$} & $7: 00-8: 00$ & 71.5 & \multirow{3}{*}{$\begin{array}{c}\text { Dia entre } \\
\text { semana }\end{array}$} & $7: 00-8: 00$ & 58.5 \\
\hline & 9:00 -10:00 & 60.3 & & 8:00 - 9:00 & 68.7 & & 8:00 - 9:00 & 59.5 \\
\hline & 10:00 - 11:00 & 62.3 & & $9: 00-10: 00$ & 69.4 & & $9: 00-10: 00$ & 60.1 \\
\hline \multirow{3}{*}{$\begin{array}{c}\text { Fin de } \\
\text { semana }\end{array}$} & $8: 00-9: 00$ & 70.4 & \multirow{3}{*}{$\begin{array}{c}\text { Fin de } \\
\text { semana }\end{array}$} & $7: 00-8: 00$ & 69.5 & \multirow{3}{*}{$\begin{array}{c}\text { Fin de } \\
\text { semana }\end{array}$} & $7: 00-8: 00$ & 70.1 \\
\hline & 9:00 -10:00 & 71.1 & & 8:00 - 9:00 & 71.5 & & $8: 00-9: 00$ & 68.1 \\
\hline & 10:00 - 11:00 & 70.5 & & $9: 00-10: 00$ & 68.6 & & $9: 00-10: 00$ & 67.1 \\
\hline
\end{tabular}

Fuente: elaboración propia.

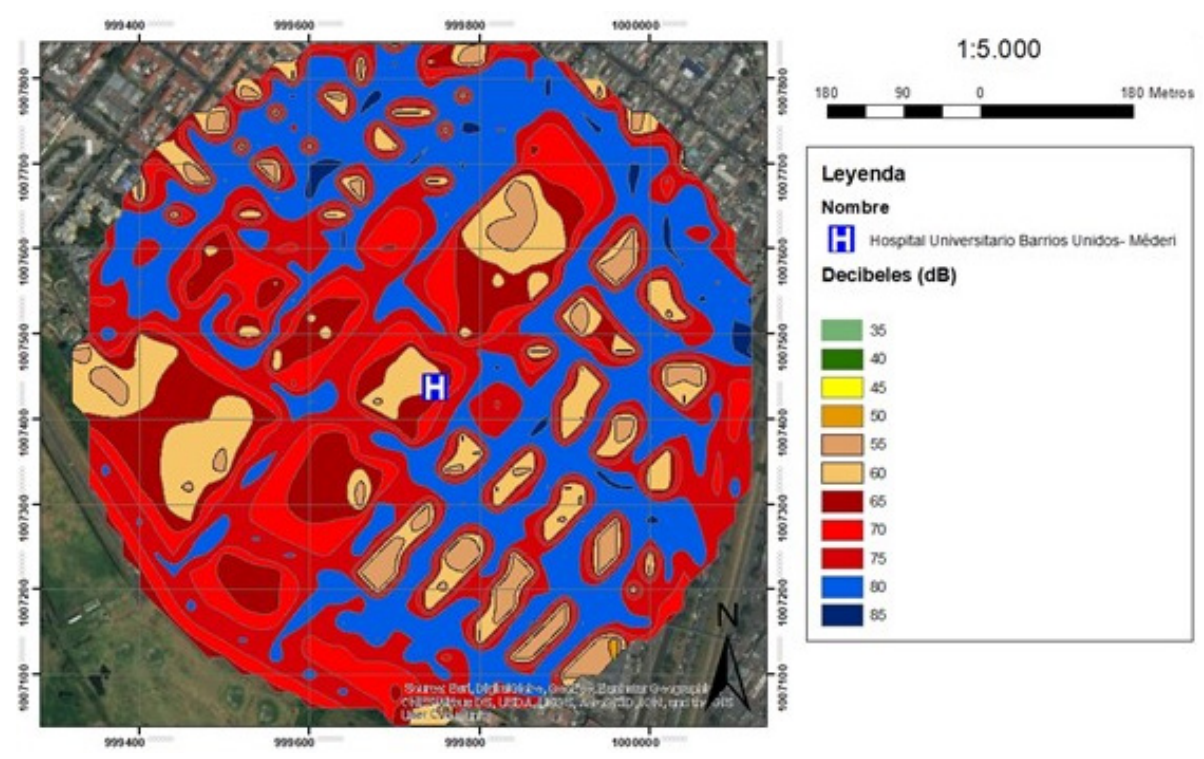

Figura 4. Mapa de distribución de ruido en el Hospital Universitario Barrios Unidos.

Fuente: elaboración propia.

incluso hasta $80 \mathrm{~dB}$; mientras que las áreas sombreadas con color rojo dan respuesta a la ubicación de fuentes fijas como lo son parqueaderos, colegios y parques donde se presenta un alto nivel de ruido. Las áreas que presentan menores niveles de ruido están alrededor de las edificaciones, en lugares que se comportan como barreras con relación a la ubicación de las principales actividades fuente de ruido.

\section{Mapa de distribución CAPS de Chapinero}

Realizando un análisis de los datos obtenidos, y el modelo realizado, se puede observar que, en el CAPS de Chapinero los NPS predominantes en esta zona se encuentran en un intervalo entre 75 $\mathrm{dB}$ en sitios en donde predominan fuentes fijas de ruido y de hasta $80 \mathrm{~dB}$ en vías de circulación de tráfico automotor. Las dos fuentes fijas ubicadas al 
frente del centro médico (taller de metalurgia y paradero del SITP), presentan datos en un intervalo desde $70 \mathrm{~dB}$ hasta $80 \mathrm{~dB}$. En el mapa (figura 5) se observan áreas de bajo nivel de ruido debido a la atenuación presentada por las mismas barreras de las edificaciones.

\section{Mapa de distribución de ruido en la Fundación Hospital Infantil Universitario de San José}

La propagación del ruido en la zona del Hospital infantil (figura 6) permite definir que la fuente generadora de ruido más importante es el alto flujo vehicular, dada la cercanía a avenidas principales. Además, se comprobó en las mediciones realizadas que el valor de L90, que da una descripción del ruido de fondo, todo el tiempo fue mayor de 55 $\mathrm{dB}$. El intervalo en decibeles observado en la zona aledaña al centro médico (65 dB a $70 \mathrm{~dB}$ ) tiene una alta influencia por las vías cercanas en donde se encuentra un nivel de ruido mayor (80 dB). En esta área no hay edificaciones o parques que puedan actuar como barreras y mitiguen la propagación del ruido.
En relación con las isófonas elaboradas se demuestra que la zona alrededor de los centros médicos de estudio, se presenta incumplimiento normativo con respecto al ruido ambiental y esto dado principalmente por la densidad de tráfico vehicular, lo que es característico de ambientes urbanos modernos tanto en países desarrollados como en desarrollo (McMichael, 2000). Además, en las zonas aledañas las actividades comerciales formales e informales también se manifiestan como de alta importancia ya que se mantienen niveles de ruido sobre $60 \mathrm{~dB}$ y $65 \mathrm{~dB}$, que según la Organización Mundial de la Salud a estos valores se pueden sufrir malestares fuertes, dolores de cabeza y, en pacientes dentro del entorno hospitalario, interupción de la tranquilidad y trastorno del sueño y descanso.

Las diferentes zonas de estudio, de acuerdo con el plan de ordenamiento territorial de Bogotá, son catalogadas como zonas residenciales con delimitación de comercio y servicio. Sin embargo, de manera general se evidencia que hay un desarrollo comercial alrededor y cerca de los centros médicos; en algunos casos asociado al mismo servicio

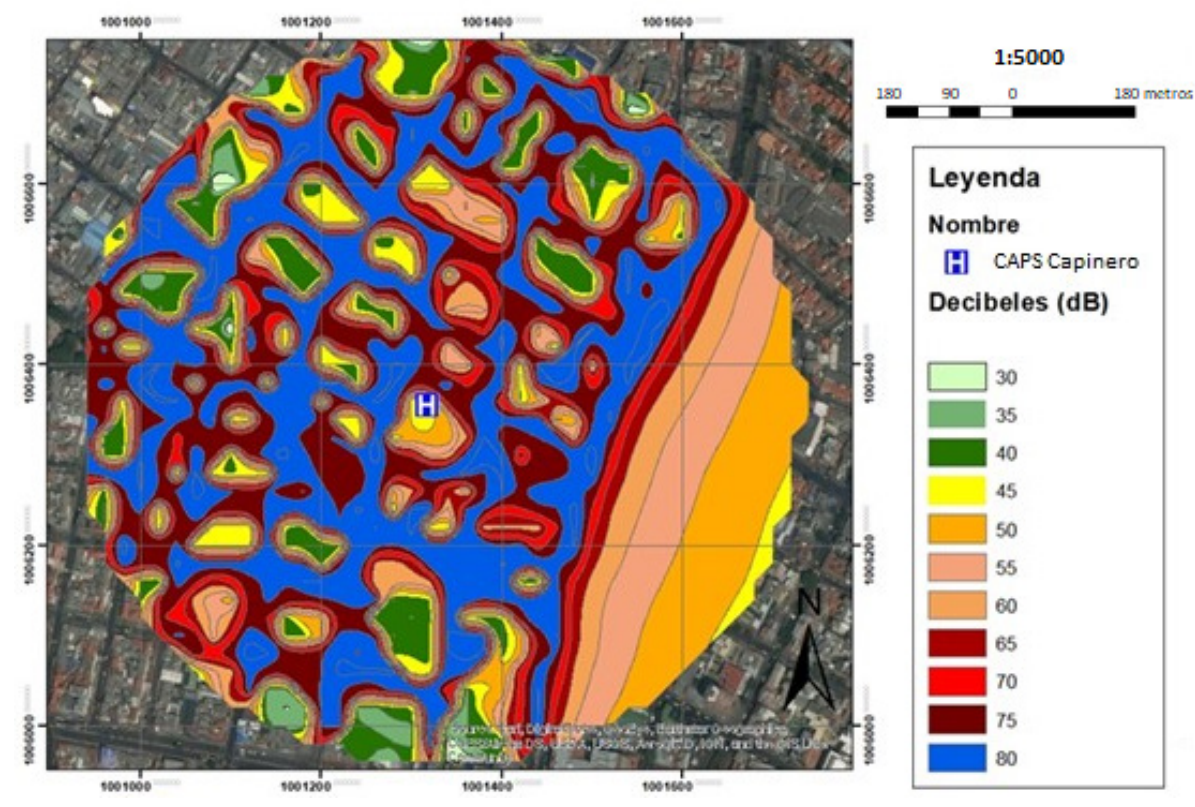

Figura 5. Mapa de distribución de ruido CAPS de Chapinero.

Fuente: elaboración propia. 


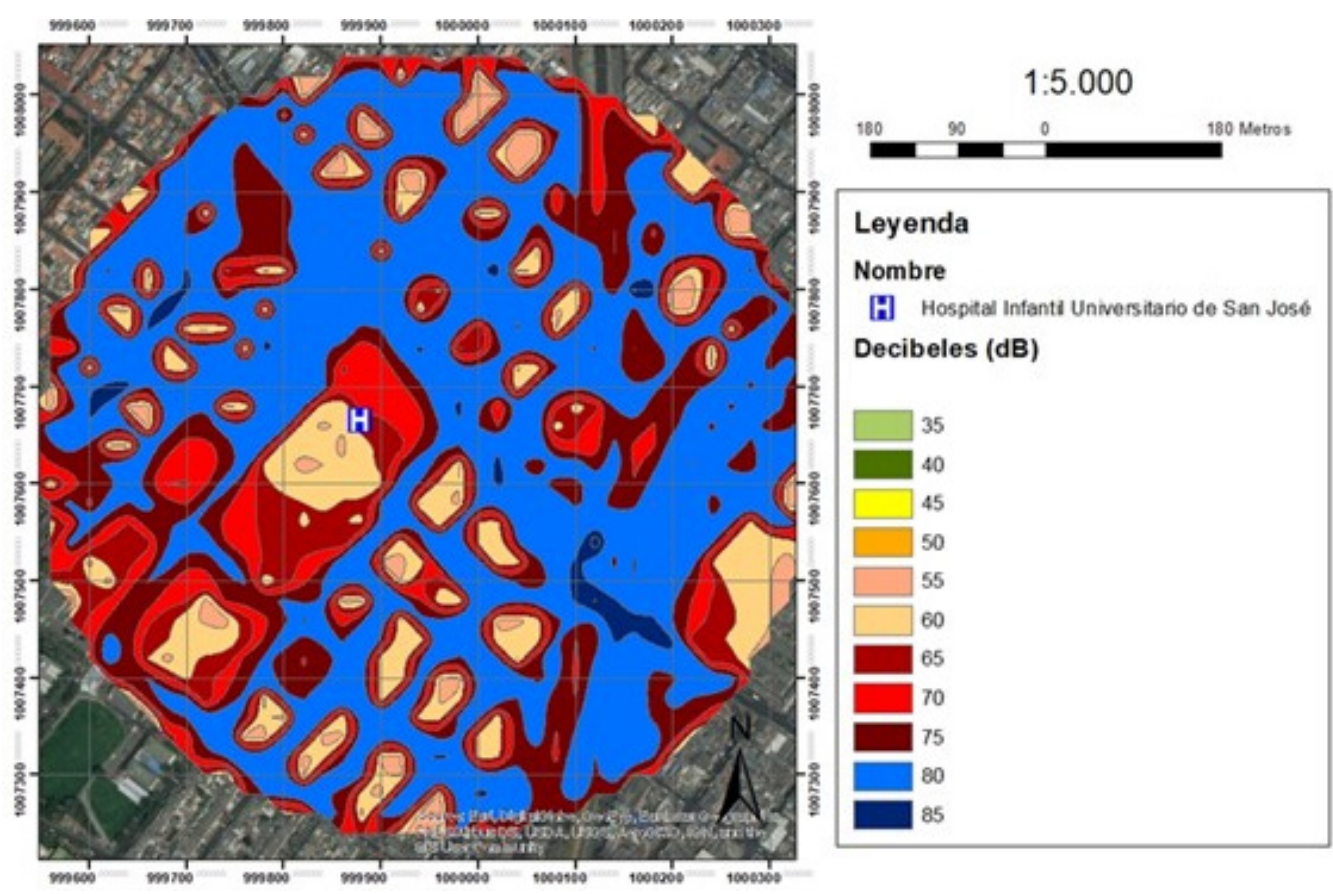

Figura 6. Mapa de distribución de ruido en la Fundación Hospital Infantil Universitario de San José.

Fuente: elaboración propia.

hospitalario (droguerías y misceláneas) y otros no asociados tales como restaurantes, panaderías, cafeterías, supermercados y ferreterías; en otros casos corresponden a actividades no autorizados o de comercio informal ventas ambulantes de gran variedad de artículos. En cuanto al tráfico vehicular, la administración distrital ha autorizado la ubicación y puesta en funcionamiento de paraderos del SITP, o al frente o en sitios muy cercanos a los centros médicos, sin tener en cuenta gestión estratégica para la contaminación por ruido.

De esta manera, ante el incumplimiento normativo con relación a la contaminación por ruido en los sectores de tranquilidad y silencio, los planes de acción que se pueden establecer se remiten principalmente a la vigilancia de cumplimiento de ordenamiento del territorio y desarrollo de políticas del crecimiento en estos sectores de la localidad de Barrios Unidos bajo lineamientos de prevención de contaminación. En algunos estudios se sugieren acciones tales como el desarrollo de transporte subterráneo y construcción de barreras de ruido que no vayan en contra de la estética urbanística (Murphy y King, 2011). Para este caso en particular, la medida de transporte subterráneo puede aplicar para los corredores de vías principales cercanas a la Fundación Hospital Infantil Universitario de San José y del Hospital Barrios Unidos, mientras que en la zona del CAPS de Chapinero pueden gestionarse actividades de ubicación más adecuada de paraderos de transporte y de actividades comerciales $y$, posteriormente, estudiar la posibilidad de construcción de barreras de ruido. Con respecto a la administración local, se puede realizar seguimiento de una planeación urbana efectiva y con participación comunitaria, aplicando criterios de prevención y atendiendo al interés común de los sectores de la localidad, lo cual puede reducir considerablemente este tipo de contaminación y, además, la contaminación correlacionada con agentes químicos atmosféricos propios del tráfico rodado (Can, et al., 2011; Parlamento Europeo, 2002). 


\section{Conclusiones}

A través de este estudio se pudo definir un diagnóstico correlacionado con centros médicos ubicados en la localidad de Barrios Unidos en Bogotá, en la que se halló e identificó que las principales fuentes de ruido son debidas principalmente a la densidad de tráfico vehicular $y$, en segundo lugar, a actividades de comercio informal. Todo ello generando incumplimiento normativo con respecto a la Resolución n. 627 del 2006 del MAVDT y con relación a los niveles de presión sonora en las áreas circundantes a estas instituciones que están enmarcadas dentro de sectores de tranquilidad y silencio. Los mapas de distribución de ruido permiten identificar las zonas aledañas a los centros médicos en intervalos entre $60 \mathrm{~dB}$ y $80 \mathrm{~dB}$, los cuales tienen actividades asociadas principalmente al tráfico vehicular con la potencialidad de generar en las personas malestares fuertes y en los pacientes interrupción del sueño y de la tranquilidad.

El crecimiento de actividades, principalmente de comercio informal y formal, así como de transporte urbano ha generado un problema de calidad del aire relacionada con ruido. De acuerdo con el Plan de Ordenamiento Territorial, el cual reglamenta el uso del suelo para la ciudad de Bogotá, se evidencia que no hay un crecimiento urbano adecuado que dé cumplimiento a lo establecido; debido a que se ha permitido: la proliferación de actividades comerciales autorizadas, la apropiación de zonas aledañas a los centros médicos para el desarrollo de actividades comerciales informales y, por parte de la administración distrital, se ha autorizado además el establecimiento de paraderos SITP en las zonas aledañas. Se denota también que por parte de la administración local y respecto a infraestructura no existe gestión para construcción de vías secundarias alternativas que permitan descongestionar las vías de acceso al hospital, o gestión hacia el seguimiento, estructuración y vigilancia efectiva de cumplimiento de políticas de prevención de contaminación por ruido.

\section{Referencias}

Abbasi, A. M.; Motamedzade, M.; Aliabadi, M.; Golmohammadi, R.; Tapak, L. (2018). Study of the physiological and mental health effects caused by exposure to low-frequency noise in a simulated control room. Building Acoustics, 25(3), 1351010X18779518. http://doi. org/10.1177/1351010x18779518

Acevedo, G. (2011). Aumenta el ruido en los hospitales. Revista Mundo HVACR, https:// www.mundohvacr.com.mx/mundo/2011/11/ aumenta-el-ruido-en-los-hospitales/

Alcaldia Mayor de Bogotá (2014). Bogotá mejor para todos. http://www.bogota.gov.co/ article/conozca-los-puntos-cr\%C3\%ADticos-por-causa-del-ruido-en-la-capital

Alonso, A. (2003). Contaminación acústica y salud. Observatorio Medioambiental, 6, 73-95.

Arana, M.; García, A. (1998). A social survey on the effects of environmental noise on the residents of Pamplona, Spain. Applied Acoustics, 53(4), 245-253. https://doi.org/10.1016/ $\underline{\text { S0003-682X(97)00067-4 }}$

Can, A. et al. (2011). Correlation analysis of noise and ultrafine particle counts in a street canyon. Science of the Total Environment, 409, 564-572. https://doi.org/10.1016/j. scitotenv.2010.10.037

Committee on Environmental Health (1997). Noise: a hazard for the fetus and newborn. Pediatrics, 100(4), 724-727. https://doi.org/10.1542/ peds.100.4.724

Davis Instruments (2002). Módulo de sensores integrados manual de instalación. Hayward: Davis Instruments Corp.

De Barrios Unidos, A. L. (2017). Plan Ambiental Local 2017-2020. Bogotá: Alcaldía Local de Barrios Unidos. https://tinyurl.com/y47concn

De España, G. (19 de octubre de 2007). Real Decreto $1367 / 2007$, por el que se desarroIla la Ley 37/2003, de 17 de noviembre, del Ruido, en lo referente a zonificación acústica, objetivos de calidad y emisiones 
acústicas. BOE, 254. http://www.boe.es/ buscar/act.php

Evans, G. W.; Lercher, P.; Meis, M.; Ising, H.; Kofler, W. W. (2001). Community noise exposure and stress in children. The Journal of the Acoustical Society of America, 109(3), 10231027. https://doi.org/10.1121/1.1340642

Gutiérrez, F. F. (2011). Estudio general de la contaminación acústica en las ciudades de Andalucía. Cuadernos geográficos de la Universidad de Granada, 49(2), 55-94.

ISO (2016). 1996-1. Acoustics-Description, measurement and assessment of environmental noise - Part 1: Basic quantities and assessment procedures.

Jones, K. (2010). Environmental Noise and Health: A review.

Loupa, G., Katikaridis, A., Karali, D. y Rapsomanikis, S. (2019). Mapping the noise in a Greek general hospital. Science of The Total Environment, 646, 923-929. https://doi.org/10.1016/j. scitotenv.2018.07.315

McMichael, A. J. (2000) The urban environment and health in a world of increasing globalization: issues for developing countries. Bull World Health Organ, 78(9), 1117-1126.

Ministerio de Ambiente, Vivienda y Desarrollo Territorial (2006). Resolución $n .^{\circ}$ 0627. Norma nacional de emisión de ruido y ruido ambiental. Bogotá: Ministerio de Ambiente, Vivienda y Desarrollo Territorial.

Miyara, F. (1999). Acústica, y sistemas de sonido. Argentina: Editorial Universidad Nacional de Rosario.

Murphy, E., King, E. A. (2011). Scenario analysis and noise action planning: Modelling the impact of mitigation measures on population exposure. Applied Acoustics, 72, 487-494. https://doi.org/10.1016/j.apacoust.2010.10.006

Murphy, E.; King, E. A.; Rice, H. J. (2009). Estimating human exposure to transport noise in central Dublin, Ireland. Environment international, 35(2), 298-302. https://doi.org/10.1016/j. envint.2008.07.026

Organización Mundial de la Salud (2008). Guidelines for community noise. World Health Organization, Geneova: Organización Mundial de la Salud.

Pacheco, J., Franco, J. F.; Behrentz, E. (2009). Caracterización de los niveles de contaminación auditiva en Bogotá: Estudio piloto. Revista de ingeniería, 30, 72-79.

Parlamento Europeo (junio 25 de 2002). Directiva 2002/49/EC. Parlamento Europeo.

Secretaría Distrital de Ambiente (2009). Agenda Ambiental Localidad 12 Barrios Unidos. https:// riosalitre.files.wordpress.com/2009/04/12 aa -barrios-unidos.pdf

Secretaría Distrital de Ambiente (s. f.). Bogotá mejor para todos. http://ambientebogota.gov.co/ruido

Secretaría Distrital de Planeación (2011). Secretaría de planeación. http://www.sdp.gov.co/ portal/page/portal/PortalSDP/InformacionTomaDecisiones/Estadisticas/Documentos/ An\%E1 lisis/DICE074-MonografiaBarriosUnidos-31122011.pdf .

Stassen, K. R., Collier, P. y Torfs, R. (2008). Environmental burden of disease due to transportation noise in Flanders (Belgium). Transportation Research Part D: Transport and Environment, 13(6), 355-358. https://doi.org/10.1016/j. trd.2008.04.003 